\title{
Malleability of Spike-Timing-Dependent Plasticity at the CA3-CA1 Synapse
}

\author{
Gayle M. Wittenberg and Samuel S.-H. Wang \\ Department of Molecular Biology and Program in Neuroscience, Princeton University, Princeton, New Jersey 08544
}

The magnitude and direction of synaptic plasticity can be determined by the precise timing of presynaptic and postsynaptic action potentials on a millisecond timescale. In vivo, however, neural activity has structure on longer timescales. Here we show that plasticity at the CA3-CA1 synapse depends strongly on parameters other than millisecond spike timing. As a result, the notion that a single spiketiming-dependent plasticity (STDP) rule alone can fully describe the mapping between neural activity and synapse strength is invalid. We have begun to explore the influence of additional behaviorally relevant activity parameters on STDP and found conditions under which underlying spike-timing-dependent rules for potentiation and depression can be separated from one another. Potentiation requires postsynaptic burst firing at $5 \mathrm{~Hz}$ or higher, a firing pattern that occurs during the theta rhythm. Potentiation is measurable after only tens of presynaptic-before-postsynaptic pairings. Depression requires hundreds of pairings but has less stringent long timescale requirements and broad timing dependence. By varying these parameters, we obtain STDP curves that are long-term potentiation only, bidirectional, or long-term depression only. This expanded description of the CA3-CA1 learning rule reconciles apparent contradictions between spike-timing-dependent plasticity and previous work at CA3-CA1 synapses.

Key words: hippocampus; CA1; LTP; LTD; bidirectional plasticity; cesium

\section{Introduction}

Synaptic learning rules describe how patterns of neural activity drive changes in synapse strength, known as synaptic plasticity. Synaptic plasticity has been observed in a wide variety of synapses and can take the form of both long-term potentiation (LTP) and long-term depression (LTD). These processes are thought to provide a substrate for the initial stages of learning and memory (Hebb, 1949).

Over the past decade, a new formalism for describing synaptic learning rules, spike-timing-dependent plasticity (STDP), has emerged from the observation that the direction of synaptic change can be determined by the precise timing of action potentials (Levy and Steward, 1983; Gustafsson et al., 1987; Bell et al., 1997; Markram et al., 1997). In the most commonly observed form of STDP between excitatory neocortical neurons, pairing presynaptic action potentials with postsynaptic action potentials occurring slightly later leads to potentiation, whereas pairing in the converse order leads to depression (Bi and Poo, 1998; Feldman, 2000; Sjöström et al., 2001; Froemke and Dan, 2002) [for other rules in nonpyramidal neurons, see Bell et al. (1997); Egger et al., 1999; Wang et al., 2000]. A measurement of the magnitude of plasticity as a function of spike timing has been taken as a

Received Dec. 16, 2005; revised May 5, 2006; accepted May 8, 2006.

This work was supported by a Princeton Council on Science and Technology fellowship (G.M.W.), National Science Foundation Grant IBN-0347719, National Institutes of Health Grant NS045193, and the W. M. Keck Foundation (S.S.-H.W.). We thank Mark Schnitzer, John Hopfield, Daniel O'Connor, Henry Abarbanel, and Carlos Brody for useful discussions.

Correspondence should be addressed to Dr. Samuel S.-H. Wang, Department of Molecular Biology, Lewis Thomas Laboratory, Washington Road, Princeton, NJ 08544. E-mail: sswang@princeton.edu.

DOI:10.1523/JNEUROSCI.5388-05.2006

Copyright $\odot 2006$ Society for Neuroscience $\quad$ 0270-6474/06/266610-08\$15.00/0 description of an impulse response of plasticity to a given pairing of presynaptic and postsynaptic activity (Song et al., 2000; Rubin et al., 2001). However, the complete description of any neural activity pattern lies in a high-dimensional space, in which spike timing is only one of the dimensions.

Induction of both LTP and LTD requires the elevation of postsynaptic calcium. However, the two processes are evoked through different calcium-dependent signal transduction cascades, with LTP requiring kinase activation (Malenka et al., 1989; Malinow et al., 1989) and LTD requiring phosphatase activation (Mulkey et al., 1993). If each pathway has a different sensitivity to activity parameters beyond spike timing, multiple STDP rules will be measurable at a single synapse.

For this study, we have chosen to examine the hippocampal CA3-CA1 synapse in acutely prepared brain slices. In this preparation, $>20$ years of experiments have provided a rich source of information about synaptic plasticity and its underlying molecular mechanisms (Bliss and Collingridge, 1993; Malenka and Nicoll, 1999; Sanes and Lichtman, 1999). However, reports of STDP have been inconsistent with both each other and previous work at this synapse. By trying to understand dimensions of the learning rule beyond spike-timing dependence, our aim is to reconcile these bodies of work and, in doing so, to better understand the role of spike-timing dependence in determining synapse strength.

Our chosen parameter space was motivated by known in vivo activity patterns in the hippocampus, for which a rich literature exists. We measured the spike-timing dependence of plasticity while varying the pairing frequency, number of pairings, and postsynaptic firing mode. Subject to variation of these parameters, we find the STDP rule for this synapse to be quite malleable. In conditions that satisfy the requirements for depression, but 
not potentiation, we observe an LTD-only timing curve. Conversely, by satisfying requirements for potentiation, but not depression, we observe an LTP-only timing curve. Our results indicate the existence of two general rules, one for potentiation and one for depression, that can account for a wide range of previous observations.

\section{Materials and Methods}

Slice preparation. Transverse hippocampal slices were prepared from 14to 21-d-old Sprague Dawley rats. Isoflurane-anesthetized rats were decapitated in ice-cold artificial CSF (ACSF) bubbled to equilibrium with $95 \% \mathrm{O}_{2} / 5 \% \mathrm{CO}_{2}$. Slices $(300 \mu \mathrm{m})$ were cut using a Leica (Nussloch, Germany) VT1000 S vibratome, preincubated at $34^{\circ} \mathrm{C}$ for $10-20 \mathrm{~min}$, and then transferred to an interface chamber at room temperature until use within 1-8 $\mathrm{h}$ after slice preparation. ACSF for slicing and recording contained the following (in $\mathrm{mM}$ ): $126 \mathrm{NaCl}, 3 \mathrm{KCl}, 1 \mathrm{NaH}_{2} \mathrm{PO}_{4}, 2 \mathrm{CaCl}_{2}$, 1 or $2 \mathrm{MgCl}_{2}, 25 \mathrm{NaHCO}_{3}$, and 25 D-glucose (320 mOsm, pH 7.3). In indicated control experiments, $100 \mu \mathrm{m}$ picrotoxin was added to the recording ACSF, and a cut was made to remove area CA3 from the slice to prevent epileptiform activity.

Electrophysiology. Slices were transferred to a submersion-type recording chamber (Warner Instruments, Hamden, CT), secured beneath a nylon harp, and perfused $(2-4 \mathrm{ml} / \mathrm{min})$ with ACSF heated to $24-28^{\circ} \mathrm{C}$ or, in eight control experiments, to $30-34^{\circ} \mathrm{C}$. Whole-cell patch-clamp recordings were established using the blind patch method (Blanton et al., 1989). Recording electrodes were made from borosilicate glass, pulled to a resistance of 2.5-5.5 M , and filled with intracellular solution containing the following (in mM): 133 methanesulfonic acid, $7.4 \mathrm{KCl}, 0.3 \mathrm{MgCl}_{2}$, $3 \mathrm{Na}_{2} \mathrm{ATP}, 0.3 \mathrm{Na}_{2} \mathrm{GTP}$, and 10 NaHEPES, pH to 7.3 with $\mathrm{KOH}(290$ $\mathrm{mOsm}$ ). Cesium-based intracellular solution contained the following (in mм): 133 Cs-methanesulfonate, $7.4 \mathrm{CsCl}, 0.3 \mathrm{MgCl}_{2}, 3 \mathrm{Na}_{2} \mathrm{ATP}, 0.3$ $\mathrm{Na}_{2} \mathrm{GTP}$, and $10 \mathrm{NaHEPES}$, pH to 7.3 with $\mathrm{HCl}$.

A stainless steel concentric bipolar stimulating electrode of $150 \mu \mathrm{m}$ diameter (FHC, Bowdoinham, ME) was positioned in stratum radiatum near CA3. Cells were held in voltage clamp at $-70 \mathrm{mV}$ except for eight recordings done in current clamp. EPSCs were evoked either every 10 or $30 \mathrm{~s}$. The stimulation intensity was adjusted until EPSC responses on screen were judged to be between 50 and $150 \mathrm{pA}(92 \pm 36 \mathrm{pA}$, mean \pm $\mathrm{SD}$ ), of order 10 times as large as unitary CA3-CA1 synaptic strength (O'Connor et al., 2005a). Based on a $5 \mathrm{mV}$ hyperpolarizing voltage test step during each sweep, the input resistance was $190 \pm 49 \mathrm{M} \Omega$ (mean \pm $\mathrm{SD}$ ), and the series resistance, which was not compensated in voltage clamp recordings, was $32 \pm 16 \mathrm{M} \Omega$ (mean $\pm \mathrm{SD}$ ). All recordings were switched to current clamp for the pairing period. Resting membrane potentials were typically between -55 and $-68 \mathrm{mV}$. Action potentials were evoked via short depolarizing pulses (1.2-2 nA, $3 \mathrm{~ms}$ ). Doublets were evoked by either a single prolonged current injection $(20-30 \mathrm{~ms}$; $n=5$ ) or by two $3 \mathrm{~ms}$ current steps initiated $10 \mathrm{~ms}$ apart from one another $(n=67)$. The interval between the two action potential times over all experiments was $10.3 \pm 0.9 \mathrm{~ms}$ (mean $\pm \mathrm{SD}$ ).

Data acquisition and analysis. Signals were amplified with a Multiclamp 700A amplifier (Molecular Devices, Palo Alto, CA), acquired using either a Digidata 1320A and pClamp 8 software (Molecular Devices) or an NI-6052E (National Instruments, Austin, TX) and custom Matlab (MathWorks, Natick, MA) software, filtered at $2 \mathrm{kHz}$, and digitized at 10 kHz. Data analysis was performed using both Clampfit 8.1 (Molecular Devices) and Matlab 7.0 (MathWorks).

In voltage-clamp recordings, the amplitude of the EPSC was measured as the average over a $1.5 \mathrm{~ms}$ window at the EPSC peak. In current-clamp recordings, the initial slope of the EPSP slope was measured during the initial $2 \mathrm{~ms}$ after EPSP onset. These quantities were recorded for every sweep. The first $10 \mathrm{~min}$ of recording before the pairing protocol were used to calculate baseline responses except in two experiments in which series and input resistance had not yet stabilized and only the last 5-8 min of the baseline period was used.

For plasticity protocols, timings are reported as the difference between EPSP onset and action potential peak. For protocols in which an action potential doublet was evoked, times are reported relative to the later of two action potentials. The amount of plasticity reported for each exper- iment shown in the three spike-timing-dependent plasticity curves is the normalized change in response size during the 10-20 min period after the plasticity protocol. In a small number of experiments ending before this time, the last $10 \mathrm{~min}$ of the recording were used. Experiments were discarded if input or series resistance measured during this period differed from baseline values by $>30 \%$. Unless otherwise indicated, uncertainty is reported as \pm SEM and significance tests are one-tailed $t$ tests.

\section{Results}

We recorded synaptic responses from acutely prepared hippocampal brain slices in 108 whole-cell recordings from CA1 pyramidal neurons (Fig. $1 A$ ). In these recordings, we controlled presynaptic activation of the Schaffer collaterals using an extracellular stimulating electrode (S) placed in the stratum radiatum. Postsynaptic action potentials were generated in current-clamp recording mode by injecting 1.2-2 nA of current for $3 \mathrm{~ms}$ through the patch pipette. To induce synaptic plasticity, we paired presynaptic stimuli with postsynaptic action potentials under various timing conditions.

\section{Conditions for a depression-only learning rule}

STDP at CA3-CA1 synapses has not been a robust phenomenon. In different laboratories, causal pairings of single presynaptic and postsynaptic action potentials at these synapses lead to LTP (Nishiyama et al., 2000), to no plasticity (Pike et al., 1999), and even to LTD (Christie et al., 1996). We therefore began by establishing the effect of a widely used STDP induction protocol. We paired action potentials in causal order (Fig. $1 B$ ), with the presynaptically triggered EPSP onset occurring between 0 and $20 \mathrm{~ms}$ before the peak of the postsynaptic action potential. In notable contrast to the effect of similar protocols at other excitatory synapses, LTP was not observed. Potentiation was not seen under a variety of conditions, including 70-100 causal (pre before post) pairings given at low $(0.1-0.5 \mathrm{~Hz})$ or high $(5 \mathrm{~Hz})$ frequencies and, in one experiment (shown in Fig. $1 B$ ), 200 pairings given at $5 \mathrm{~Hz}$. Across experiments, the mean response was not potentiation but a weak depression (Fig. $1 B$ ) (averaged time course; synaptic strength, $0.86 \pm 0.08$ times the pre-pairing baseline, $n=8$; not greater than $1, p=0.95)$. In addition, potentiation was not observed at high temperatures $\left(30-34^{\circ} \mathrm{C}\right)$ with 100 pairings given at $5 \mathrm{~Hz}(0.89 \pm 0.04$ times the pre-pairing baseline, $n=3$; not greater than $1, p=0.93)$. Pairing in the reverse order, with the postsynaptic action potential evoked before presynaptic stimulation within $25 \mathrm{~ms}$, gave an average response (Fig. 1C) of depression (synaptic strength, $0.81 \pm 0.08$ of baseline, $n=7 ; p<0.03$ ), consistent with measurements made at other cortical synapses.

Combining these two datasets, depression or no significant change was seen in all but 1 of 15 experiments. The average effect of pairing single presynaptic and postsynaptic action potentials within $25 \mathrm{~ms}$ of one another was LTD $(0.84 \pm 0.05$ of baseline, $n=16$, room temperature experiments only; $p<0.005$, twotailed $t$ test). In contrast, in control experiments in which pairings of EPSPs and action potentials were given $>50 \mathrm{~ms}$ apart, no change was observed $(0.99 \pm 0.02$ of baseline, $n=16 ; p=0.8$, two-tailed $t$ test), indicating that depression was specific to nearsynchronous pairings of presynaptic and postsynaptic action potentials.

These results suggested that the pairing of single presynaptic and postsynaptic action potentials might give a depression-only learning rule. We therefore measured the plasticity resulting from pairing presynaptic and postsynaptic action potentials at intervals ranging from $\Delta t=+100 \mathrm{~ms}$ (positive intervals being defined as causal) to $\Delta t=-100 \mathrm{~ms}$ (anti-causal, postsynaptic action 
a

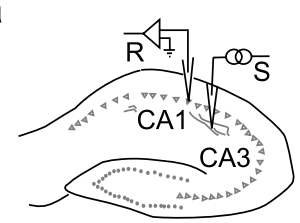

b

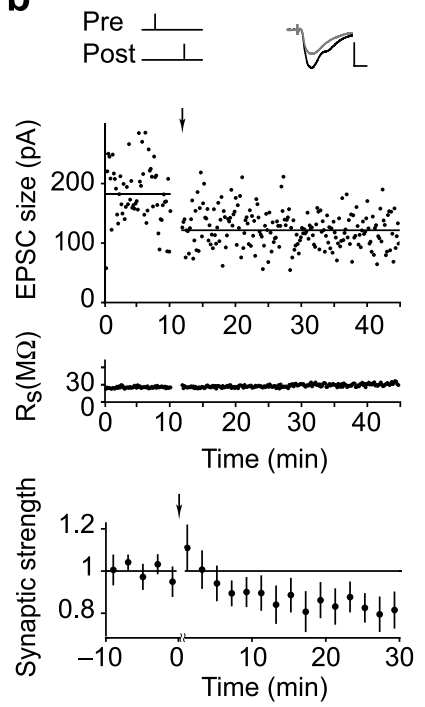

d

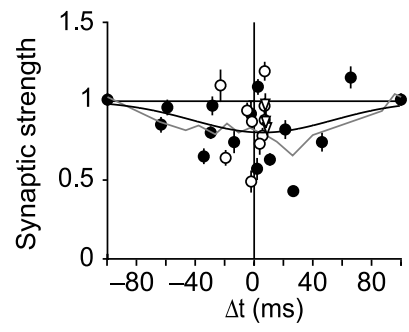

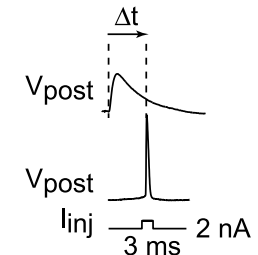

C
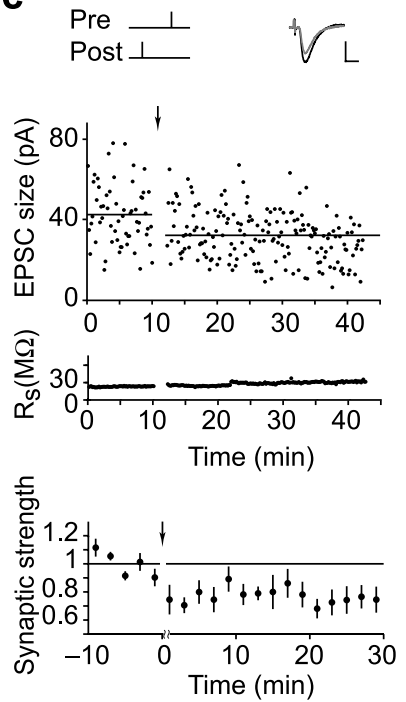

e

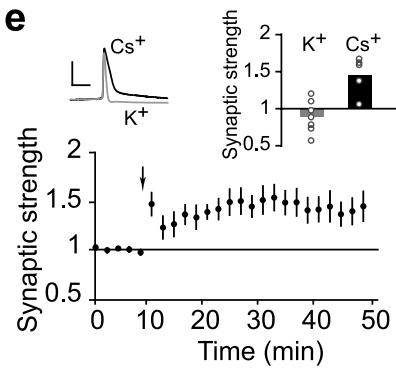

Figure 1. Pairing single presynaptic and postsynaptic action potentials leads to an LTD-only learning rule. $\boldsymbol{a}$, Experimental design. The timing of presynaptic action potential activity was controlled by a stimulating electrode (S) placed in stratum radiatum near CA3. The timing of postsynaptic action potential activity was controlled by current injection through the recording electrode $(\mathrm{R})$. The action potential timing $\Delta t$ is measured from EPSP onset to action potential peak. $\boldsymbol{b}$, Causal pairings at frequencies from 0.1 to $5 \mathrm{~Hz}$ fail to induce LTP. Top, Example experiment, 200 pairings at $5 \mathrm{~Hz}$ with $\Delta t=+10.8 \mathrm{~ms}$. Inset, EPSC traces averaged over baseline period (black) and 10-20 min after pairing (gray). Calibration: 100 pA, 10 ms. Middle, Series resistance. Bottom, Averaged data from eight experiments for which $0<\Delta t<+20 \mathrm{~ms}$. Arrows indicate the time of the plasticity protocol. The break in the axis represents the pairing period to align protocols of different frequencies and therefore different durations. c, Anti-causal pairings. Top, Example experiment, 100 pairings at $5 \mathrm{~Hz}$ with $\Delta t=-13.7 \mathrm{~ms}$. Inset, EPSC traces averaged over baseline period (black) and 10-20 min after pairing (gray). Calibration: 20 pA, $10 \mathrm{ms.}$ Middle, Series resistance. Bottom, Averaged data from seven experiments for which $-25<\Delta t<0 \mathrm{~ms}$. $\boldsymbol{d}$, Spike-timing dependence for pairings between -100 and $100 \mathrm{~ms}$. Open circles indicate pairings delivered at frequencies from 0.1 to $0.5 \mathrm{~Hz}$. Filled circles indicate experiments in which pairings were repeated at $5 \mathrm{~Hz}$. Open triangles indicate control experiments at $30-34^{\circ} \mathrm{C}$. The resulting learning rule consists of a single broad depression-only window. Error bars indicate the confidence intervals for individual experiments. Gray curve, Moving average of four data points; black curve, a Gaussian fit to the $5 \mathrm{~Hz}$ data. $\boldsymbol{e}$, Causal pairings lead to LTP in cesium-based, potassium-free intracellular solution. Top left, Action potentials recorded in potassium (gray) and cesium (black) pipette solutions. Calibration: $40 \mathrm{mV}, 10 \mathrm{~ms}$. Top right, Outcome of plasticity experiments for which $0<\Delta t<+10 \mathrm{~ms}$. The open circles indicate individual experiments, and the bar height indicates averages. Bottom, Averaged data for cesium experiments.

potential before presynaptic action potential). The resulting curve consisted of depression only (Fig. $1 D$ ) and took the form of a broadly tuned timing window that was approximately Gaussian, reaching a maximum depression to $0.80 \pm 0.05$ of baseline, centered at $\Delta t=$ $+6 \pm 28 \mathrm{~ms}$ with a half-maximal width of $113 \pm 72 \mathrm{~ms}$.

Our findings conflict with a report that pairs of single spikes induce bidirectional plasticity at CA3-CA1 synapses (Nishiyama et al., 2000). One possible reason for this discrepancy is the use by Nishiyama et al. of a patch pipette intracellular solution containing cesium instead of potassium, the normally occurring positive ion. Cesium blocks potassium channels, a manipulation that can depolarize the postsynaptic neuron, broaden action potentials, and enhance backpropagation of action potentials in the apical dendrite, in which CA3 synaptic inputs impinge (Hoffman et al., 1997). In other synapses, partial block of NMDA-type glutamate receptors converts LTP to LTD (Nishiyama et al., 2000; Froemke et al., 2005), suggesting that, conversely, a cesium-based solution could facilitate LTP induction by increasing the magnitude or time course of dendritic depolarization, thus enhancing NMDA receptor activation. We therefore made additional recordings using a cesium-based intracellular solution.

With cesium in the pipette, action potentials were broadened considerably (half-maximal width of spontaneous action potentials, $9.2 \pm 0.6 \mathrm{~ms}, n=4$ with cesium compared with $2.1 \pm 0.2$ $\mathrm{ms}, n=5$ with potassium patch solution) (Fig. $1 E$ ), the resting membrane potential was depolarized, and negative current injection was necessary to hold neurons near a normal resting potential during plasticity protocols. Under these conditions, pairing of presynaptic stimuli with single postsynaptic spikes induced LTP in each of five experiments ( $\Delta t=0$ to $+5 \mathrm{~ms} ; 1.45 \pm 0.11$, $n=5 ; p<0.01$ ) (Fig. $1 E$ ). Thus, recording with cesium may allow LTP induction in response to pairings of single spikes by causing action potentials to be nonphysiologically long.

Can the finding that causal pairings of single spikes leads to synaptic depression account for previously observed properties of LTD induced by low-frequency stimulation (LFS)? Lowfrequency extracellular stimulation of the Schaffer collaterals is a reliable and well studied protocol for the induction of homosynaptic LTD (Dudek and Bear, 1992). Typically, stimulation of presynaptic fibers $200-900$ times at frequencies ranging from 0.5 to $5 \mathrm{~Hz}$ leads to depression of whole-cell or field EPSPs. For this protocol to induce LTD, action potential activity must occur in the postsynaptic neuron (Christie et al., 1996). One would expect these postsynaptic spikes to occur primarily in causal order, immediately after presynaptic stimuli.

We recorded all postsynaptic spike activity during an LFS protocol consisting of $3.3 \mathrm{~Hz}$ stimulation for $5 \mathrm{~min}$ (900 presynaptic stimuli in $270 \mathrm{~s}$ ) (Fig. $2 A, B$ ). In these experiments, we simultaneously made field recordings near the whole-cell recording site. Before and after the LFS period, subthreshold EPSCs were measured in voltage clamp. During the LFS period, the stimulus intensity was either increased so that the field EPSP was one-half to two-thirds the maximal amplitude obtainable by turning up the stimulus (Dudek and Bear, 1992) or not changed. Both conditions resulted in evoked postsynaptic action potentials during the conditioning protocol. Across experiments, the 900 presynaptic stimuli evoked, on average, 200 postsynaptic action potentials, with delays from EPSP onset to action potential peak ranging from 1 to $25 \mathrm{~ms}(6.2 \pm 4.0 \mathrm{~ms}$, mean $\pm \mathrm{SD}$ ) (Fig. 2C). In all three experiments, this led to long-term depression of synaptic responses (average, $0.65 \pm 0.10$ of baseline, $n=3 ; p<0.05$ ) (Fig. $2 D$ ). Thus, a low-frequency stimulation paradigm for inducing long-term de- 
a

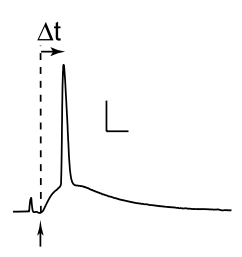

C

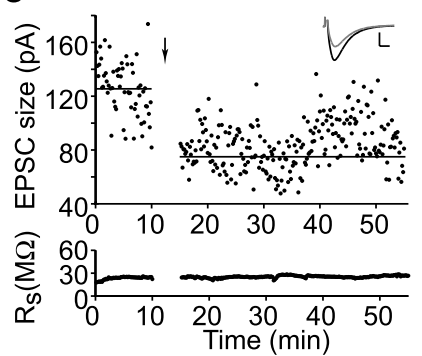

b

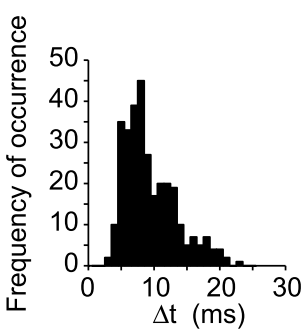

d

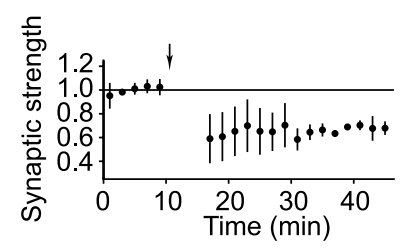

Figure 2. Low-frequency tetanic stimulation protocols for LTD induction elicit postsynaptic action potentials with causal timing. $\boldsymbol{a}$, Whole-cell recording of an action potential triggered by extracellular stimulation in current clamp. Spike timing was measured from the EPSP onset to action potential peak. Calibration: $20 \mathrm{mV}, 5 \mathrm{~ms}$. $\boldsymbol{b}$, Histogram of all spike timings recorded during conditioning, which consisted of 900 presynaptic stimuli delivered at 300 ms intervals (3.3 Hz).c, Resulting plasticity for the experiment in $\boldsymbol{b}$. Inset, EPSC traces averaged over baseline period (black) and 10-20 min after tetanus (gray). Calibration: 50 pA, 20 ms. d, Averaged data from all three experiments. The arrow indicates the time of the conditioning protocol.

pression triggers causal pairs of presynaptic and postsynaptic spikes and conforms to our single-spike plasticity rule.

\section{Conditions for a bidirectional learning rule}

Previous field recording studies indicated that LTP at CA3-CA1 synapses requires structured activity on two timescales (Larson and Lynch, 1986; Rose and Dunwiddie, 1986). First, LTP requires a short timescale associated with bursts of action potentials separated by $\sim 10$ ms (Pike et al., 1999; Watanabe et al., 2002), a condition likely to depolarize CA1 dendrites strongly (Buzsaki, 2002; Lisman and Spruston, 2005). LTP also requires a longer timescale over which activity occurs repeatedly at $200 \mathrm{~ms}$ intervals, the approximate period of the theta rhythm (Larson and Lynch, 1986; Rose and Dunwiddie, 1986; Otto et al., 1991). These requirements for potentiation have not been observed in neocortical synapses (Sjöström et al., 2001; Froemke and Dan, 2002) or in hippocampal cultures (Wang et al., 2005), indicating that some biophysical requirements for inducing bidirectional synaptic plasticity are specific to CA1 pyramidal neurons. We modified our spike-based protocol to match these requirements by replacing single postsynaptic action potentials with burst-like pairs of action potentials (doublets) spaced 10-13 ms apart.

Pairing of presynaptic stimuli with postsynaptic doublets was repeated 70-100 times at $5 \mathrm{~Hz}$. For pairings in which the EPSP onset was 10-25 ms before the second action potential $(+10<$ $\Delta t<+25 \mathrm{~ms}$ ), strong LTP $(1.58 \pm 0.11$ of baseline, $n=7 ; p<$ 0.001 ) was observed (Fig. $3 A$ ). To test whether postsynaptic bursting alone is sufficient for enabling LTP induction, we decreased the pairing frequency from 5 to $0.5 \mathrm{~Hz}$. Under this condition, LTP was not observed in any of four recordings (average, $0.80 \pm 0.08$ of baseline, $n=4$; average greater than $1, p=0.96$ ) (Fig. $3 B$ ), indicating that successive postsynaptic bursts trigger

effects lasting tenths of a second that help determine whether potentiation can occur.

In other synapses, reversing the order of pairing so that the postsynaptic action potential precedes the presynaptic action potential leads to NMDA receptor-dependent long-term depression (Bi and Poo, 1998; Abbott and Nelson, 2000; Feldman, 2000). We tested whether this was true for the high-frequency postsynaptic burst paradigm that induces LTP. We evoked postsynaptic doublets that ended 3-20 ms before the onset of the EPSP, repeated 100 times at $5 \mathrm{~Hz}$. This anti-causal protocol led to depression (average, $0.74 \pm 0.07$ of baseline, $n=6 ; p<0.01$ ) (Fig. $3 C$ ). Thus, induction of LTD by reverse pairings is possible at both low and high frequencies and with both single postsynaptic action potentials and doublets. We also observed a second, causal timing window during which repetitive pairings induce LTD. This second depression window arises when the EPSP occurs $\sim 25-50 \mathrm{~ms}$ before the onset of the postsynaptic burst (Fig. $3 D, E$ ) and results in a depression to $0.71 \pm 0.08$ of baseline $(n=6 ; p<0.01)$. LTD was also observed in this time interval in recordings at higher temperatures, $30-34^{\circ} \mathrm{C}(0.72 \pm 0.11$ of baseline, $n=5 ; p<0.05)$. The similarity of this result to that found at lower temperatures indicates that the LTP and LTD mechanisms can both be driven by causal pairings over a range of temperatures approaching physiological conditions. Thus, the learning rule measured for 100 pairings of single EPSPs with postsynaptic action potential doublets at $5 \mathrm{~Hz}$ is a bidirectional sombrero-shaped curve with timing windows for both LTP and LTD.

It has been suggested that the LTD induced by causal pairings of presynaptic and postsynaptic action potentials in the brain slice preparation may be attributable to the presence of inhibition (Nishiyama et al., 2000; Tsukada et al., 2005). However, lowfrequency field stimulation protocols are known to evoke LTD in the presence of picrotoxin (Steele and Mauk, 1999), suggesting that LTD can still be induced with causal spike pairings even when $\mathrm{GABA}_{\mathrm{A}}$ receptors are blocked. We tried to minimize the effect of feedforward inhibition in our experiments by stimulating the Schaffer collaterals far $(>0.5 \mathrm{~mm})$ from the recording site. In addition, we performed 14 experiments in the presence of picrotoxin under timing conditions that evoked causally induced LTD when no drug was present. For experiments timed with the EPSP onset 30-60 ms before the second action potential, significant $(p<0.05)$ LTD was seen in 7 of 12 experiments, significant LTP in 2 experiments, and no plasticity in 3 experiments (Fig. $3 F$ ). Overall, the magnitude of depression observed in picrotoxin was reduced compared with the control curve in this same time window (in picrotoxin, $0.88 \pm 0.06$ of baseline, $n=12$; control, $0.74 \pm 0.10$ of baseline, $n=5 ; p<0.05)$. One possible explanation for the reduced magnitude of LTD is that the ability of the first action potential in the doublet to backpropagate has been enhanced (Tsubokawa and Ross, 1996) so that one spike alone is sufficient for the induction of LTP (Meredith et al., 2003), thus leading to a larger contribution of the potentiation process to the curve or a shifted timing dependence of the potentiation processes. Thus, although $\mathrm{GABA}_{\mathrm{A}}$ receptor-mediated inhibition is not a requirement for evoking causally induced LTD, it may influence the magnitude and/or precise timing conditions under which LTD occurs.

\section{Conditions for a potentiation-only learning rule}

The depression-only timing window from pairing single action potentials is wide enough to encompass both timing windows for depression observed in the bidirectional learning rule. These multiple depression windows may reflect a single common de- 
a
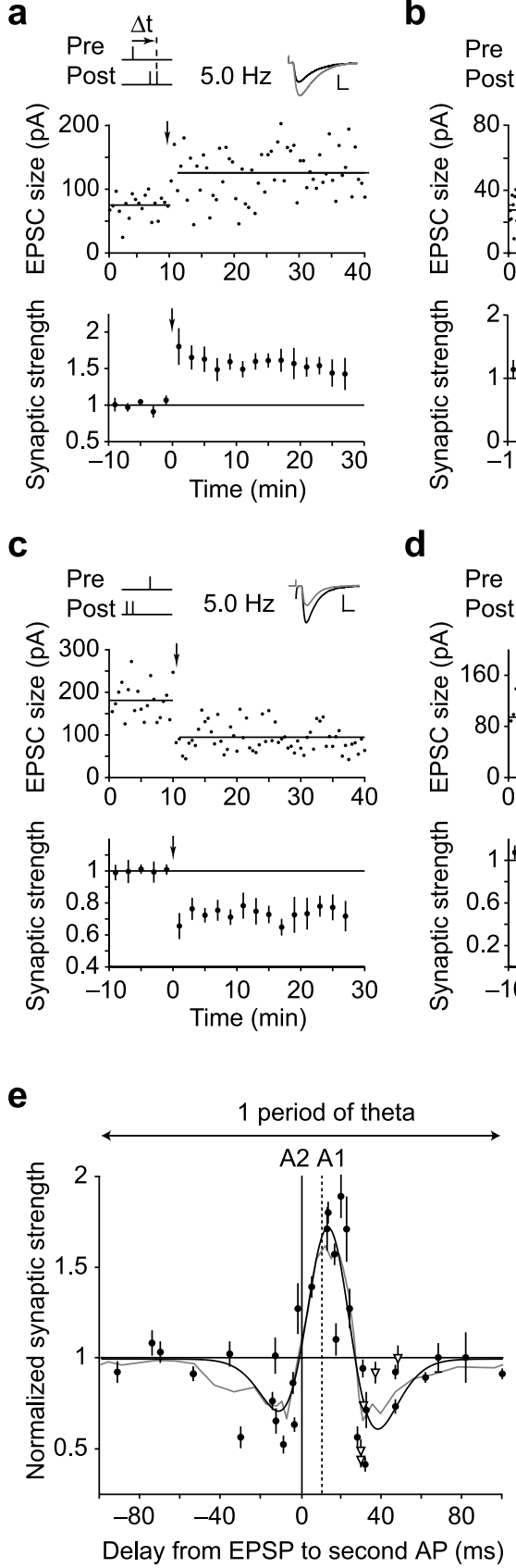

\section{f}

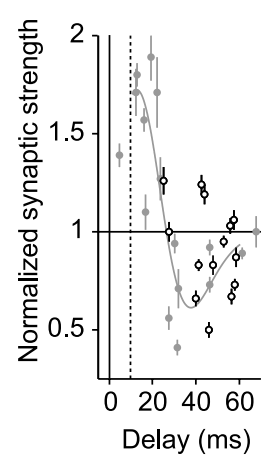

Figure 3. Pairing with postsynaptic bursts at theta frequency leads to a bidirectional learning rule. $\boldsymbol{a}$, Causal pairings near the theta frequency induce potentiation. Top, Example experiment, 100 pairings at $5 \mathrm{~Hz}$ with EPSP leading the first action potential in the burst by $9.1 \mathrm{~ms} . \Delta t$, defined as the time to the second spike, was $22.2 \mathrm{~ms}$. Inset, EPSC traces averaged over baseline period (black) and 10-20 min after pairing (gray). Calibration: 50 pA, $10 \mathrm{~ms}$. Bottom, Averaged data from seven experiments for which $+10<\Delta t<+25 \mathrm{~ms}$. Arrows indicate the time of the conditioning protocol. $\boldsymbol{b}$, Causal pairings at low frequency. Top, Example experiment, 100 pairings at $0.5 \mathrm{~Hz}$ with $\Delta t=+14.0 \mathrm{~ms}$. Inset, EPSC traces averaged over baseline period (black) and $10-20$ min after pairing (gray). Calibration: $10 \mathrm{pA}, 10 \mathrm{~ms}$. Bottom, Averaged data from four experiments for which $+10<\Delta t<+20 \mathrm{~ms}$. c, Anti-causal pairings. Top, Example experiment, 100 pairings at $5 \mathrm{~Hz}$ with $\Delta t=-9.2 \mathrm{~ms}$. Inset, EPSC traces averaged over baseline period (black) and 10-20 min after pairing (gray). Calibration: 100 pA, 10 ms. Bottom, Averaged data from six experiments for which $-20<\Delta t<-3 \mathrm{~ms}$. $d$, A second LTD window for remote causal pairings. Top, Example experiment, 100 pairings at $5 \mathrm{~Hz}$ with $\Delta t=+18 \mathrm{~ms}$. Inset, EPSC traces averaged over baseline period (black) and 10-20 min after pairing (gray). Calibration: 50 pA, $10 \mathrm{~ms}$. Bottom, Averaged data from four experiments for which $+25<$ $\Delta t<+40 \mathrm{~ms}$. $\boldsymbol{e}$, Spike-timing dependence is explored for $\Delta t$ from -100 to $100 \mathrm{~ms}$. The dashed line indicates the time of the first postsynaptic action potential in most experiments. Open triangles represent high temperature controls performed at $30-34^{\circ} \mathrm{C}$. The resulting learn- a

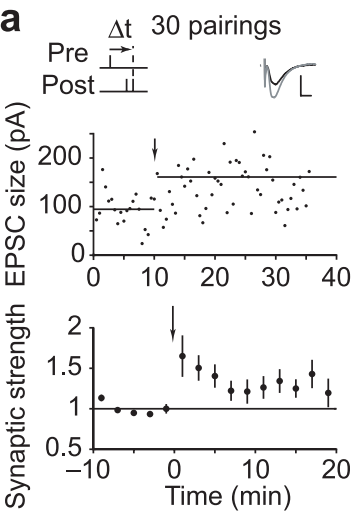

b

30 pairings

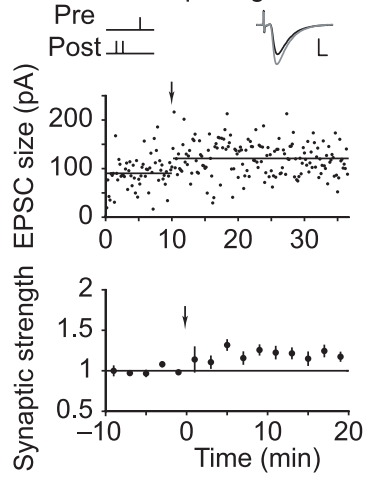

C

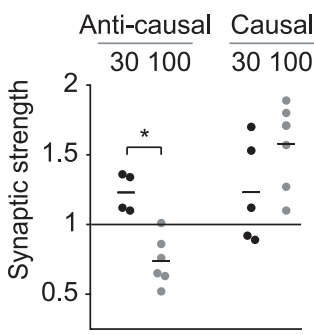

d

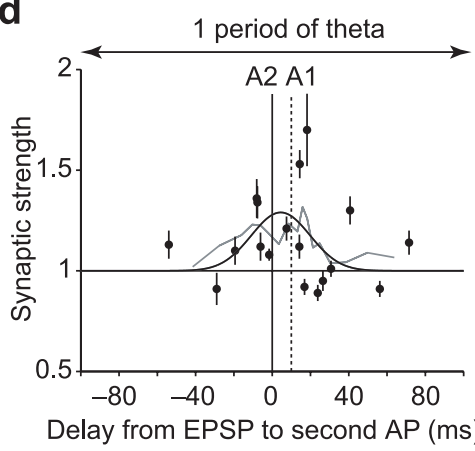

Figure 4. A potentiation-only rule for small numbers of pairings. $\boldsymbol{a}$, Causal pairings. Top, Example experiment, 20 pairings at $5 \mathrm{~Hz}$ with EPSP leading the postsynaptic burst by $8.0 \mathrm{~ms} . \Delta t$, defined as the time to the second spike, was $+18.2 \mathrm{~ms}$. Inset, EPSC traces averaged over baseline period (black) and $10-20$ min after pairing (gray). Calibration: 100 pA, $30 \mathrm{~ms}$. Bottom, Averaged data from four experiments for which $10<\Delta t<20 \mathrm{~ms}$. Arrows indicate the time of the plasticity protocol. $\boldsymbol{b}$, Anti-causal pairings. Top, Example experiment, 21 pairings at $5 \mathrm{~Hz}$ with $\Delta t=-8.1 \mathrm{~ms}$. Inset, EPSC traces averaged over baseline period (black) and $10-20 \mathrm{~min}$ after pairing (gray). Calibration: $50 \mathrm{pA}, 20 \mathrm{~ms}$. Bottom, Averaged data from five experiments for which $-20<\Delta t<0 \mathrm{~ms}$. c, Direct comparison of causal and anti-causal pairing experiments with $20-30$ ("30") or $70-100$ ("100") pairings at $5 \mathrm{~Hz} .{ }^{*} p<0.01$, Kolmogorov-Smirnov test. $\boldsymbol{d}$, Spike-timing dependence of EPSPs paired with postsynaptic bursts $20-30$ times at $5 \mathrm{~Hz}$. The dashed line indicates the time of the first postsynaptic action potential. The resulting learning rule is LTP only. The gray curve is a moving average of $n=4$ data points, anchored by averaging the two endpoints on either side. The black curve is a Gaussian fit.

pression pathway with a potentiation rule superimposed under some conditions (O'Connor et al., 2005b). This possibility led us to search for activity conditions that would give a potentiationonly learning rule.

A striking feature in protocols leading to LTD is that depression often requires activity for a longer period of time than potentiation (Dudek and Bear, 1992; Yang et al., 1999; Mizuno et al., 2001; O'Connor et al., 2005b). In addition, causal STDP protocols can lead to depression (Christie et al., 1996) but not if the number of presynaptic and postsynaptic action potential pairings is small (Pike et al., 1999; Meredith et al., 2003). Therefore, if LTP and LTD are differentially activated by the number of pairings, decreasing the number of pairings would affect the shape of the STDP curve.

When the length of the conditioning protocol was reduced from 100 pairings to $20-30$ pairings, a single LTP window was

ing rule has a narrow LTP window shifted in the causal direction flanked by two LTD windows. The gray curve is a moving average of four data points. The black curve is a fit to the sum of two Gaussians. Fits exclude high-temperature experiments. $f$, The causal portion of the LTD window measured in the presence of $100 \mu \mathrm{m}$ picrotoxin (black open circles). Gray points and gray curve represent control data from $e$ for comparison. 
observed across all timing conditions (Fig. 4). Causal pairings still led to modest potentiation ( $1.32 \pm 0.18$ of baseline, $n=5, p=$ 0.05 ; EPSP onset $0-20 \mathrm{~ms}$ before the second postsynaptic action potential) (Fig. $4 \mathrm{~A}$ ). However, pairing in the anti-causal order $(\Delta t=-20$ to $0 \mathrm{~ms}$ ) did not lead to LTD (Fig. $4 B$ ). The net synaptic change for this timing condition was, in fact, also potentiation ( $1.20 \pm 0.06$ of baseline, $n=5$; $p<0.05$ ) (Fig. $4 B$ ), in contrast with the depression seen after 100 pairings at this timing condition (compare Figs. 3C, 4C). Overall, a 30-pairing protocol results in a potentiation-only learning rule with no identifiable depression component $(1.25 \pm 0.07$ of baseline for $\Delta t=-20$ to $20 \mathrm{~ms}, n=10 ; p<0.01$, two-tailed $t$ test) (Fig. $4 D$ ). The reduced amount of LTP indicates that induction mechanisms were not saturated by the shorter protocol. Consistent with a lack of saturation, 30-pairing potentiation at $\Delta t=10-20 \mathrm{~ms}$ was more variable than 100-pairing potentiation (coefficient of variation of change from baseline, 1.13 for 30 pairings vs 0.51 for 100 pairings). From +30 to $+60 \mathrm{~ms}$ and -20 to $-60 \mathrm{~ms}$, the final synaptic strength was $1.05 \pm 0.07$ of baseline $(n=5)$, not significantly different from 1 ( $p=0.5$, two-tailed $t$ test). Overall, the potentiation-only learning rule was well fitted by a Gaussian with a peak at $1.29 \pm 0.13$ of baseline, centered at $\Delta t=+4 \pm 7 \mathrm{~ms}$ with a half-maximal width of $36 \pm 21 \mathrm{~ms}$.

\section{Comparison of component rules with the bidirectional learning rule}

To compare the properties of the potentiation and depression rules with key features of the bidirectional learning rule, we fitted the bidirectional rule with a difference of Gaussians, one for potentiation and one for depression (Fig. 3E). This fit was used to characterize the location and width of the potentiation and depression components of the rule. The depression component was centered at $\Delta t=+15 \mathrm{~ms}$, within the confidence interval of the center of the depression-only rule, $+6 \pm 28 \mathrm{~ms}$. Going outward from the troughs of depression, half-maximal values of depression occur at $\Delta t=-24$ and $+51 \mathrm{~ms}$, delineating a range of $75 \mathrm{~ms}$, comparable with the $113 \mathrm{~ms}$ width observed for the single-spike depression rule. The potentiation component was centered at $\Delta t=+13 \mathrm{~ms}$, just outside the confidence interval for the center of the potentiation-only rule at $\Delta t=+4 \pm 7 \mathrm{~ms}$ (Fig. $4 D$ ). Half-maximal values for potentiation (using the depression troughs to define a baseline) were reached over a $26 \mathrm{~ms}$ range, comparable with the $36 \mathrm{~ms}$ width of the potentiation-only rule. These comparisons indicate that the timing-dependent properties of the potentiation and depression rules change little even when both processes are activated at the same time.

\section{Discussion}

Under our recording conditions, STDP at hippocampal CA3CA1 synapses is governed by two learning rules. These rules, one for potentiation and one for depression, are distinguishable from one another not only by their dependence on precise spike timing but also on additional activity parameters that include postsynaptic bursting and the number and frequency of pairings. As a result, different STDP curves can be measured depending on the long-timescale temporal statistics of neural activity (Fig. 5). Taken as a whole, the two rules can generate a composite bidirectional learning rule and can reconcile STDP with previous results at hippocampal CA3-CA1 synapses that until now have appeared contradictory.

Our observations fit into a framework in which the mechanisms for potentiation and depression can be separated into two processes (O’Connor et al., 2005b; Wang et al., 2005). In the

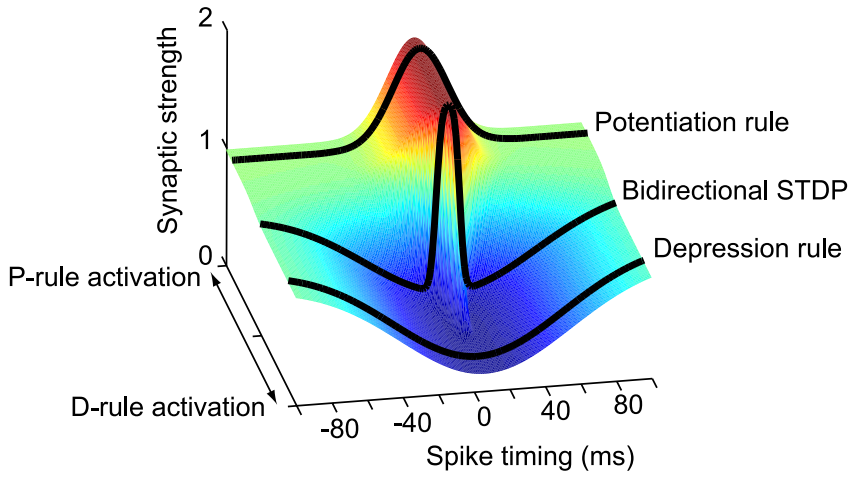

Figure 5. Spike timing is merely one dimension in the high-dimensional synaptic learning rule. A conceptual illustration of a learning rule in three dimensions is shown. Depending on the choice of activity parameters other than spike timing, many different STDP rules can be measured at the CA3-CA1 synapse. The second axis represents the transition from parameters that more strongly activate depression (D-rule) to parameters that more strongly activate potentiation (P-rule). By choosing parameters that activate only a single rule, the spike-timing dependence of LTP and LTD can be measured separately. Potentiation is initiated by as few as 20 causal pairings of presynaptic action potentials with postsynaptic bursts repeated at $5 \mathrm{~Hz}$ or higher. Depression does not require high-frequency stimulation or postsynaptic bursts but requires more pairings than LTP. Stimulus conditions that satisfy the temporal requirements for both the potentiation rule and the depression rule lead to a bidirectional spike-timing-dependent plasticity curve.

previous work, this separation was accomplished by pharmacological blockers of kinase and phosphatase signaling. Here we report that potentiation and depression depend in different ways on spike timing. Potentiation has a narrow time window shifted slightly from exact coincidence, whereas depression has a broader time window requiring only approximate coincidence. Either process has similar timing dependence to its counterpart in the composite bidirectional learning rule. The three timing windows seen for bidirectional plasticity under strong depolarization (depression, potentiation, and a second depression window) can therefore potentially be interpreted as the superposition of two processes in such a way that, when both are present, LTP wins. This is consistent with switch-like models in which, at the single synapse, plasticity is the end result of a competition between kinase- and phosphatase-dependent processes (Lisman et al., 2002) and changes in strength are all-or-none (O'Connor et al., 2005a).

Past difficulties in inducing CA3-CA1 LTP with spike-timingbased protocols have been disconcerting given the preeminence of these synapses as a model system for studying synaptic plasticity. Our results suggest that these difficulties stem from a requirement that, for LTP to be induced, CA1 dendrites must be activated fully and persistently (Pike et al., 1999; Watanabe et al., 2002). We interpret the doublets used in this study as a minimal burst, a level of postsynaptic activation for LTP induction that subsumes larger depolarizations. In vitro, synaptically induced spike bursts correspond to regenerative electrical events in the apical dendrite of pyramidal neurons (Stuart et al., 1997; Williams and Stuart, 1999). Such a requirement for dendritic activation would be met in vivo during the theta rhythm (Otto et al., 1991; Paulsen and Sejnowski, 2000; Buzsaki, 2002), which occurs during active exploration.

Our observed rules for potentiation and depression are consistent with the view that hippocampal synaptic plasticity can reinforce brief, rapid activity sequences while weakening nonspecific activity sequences spread over longer timescales. The potentiation rule requires as few as 20 pairings, suggesting that potentiation can be triggered during a few seconds of theta oscillation. 
Consistent with this feature of LTP, experience-dependent expansion of a place field is measurable after just a few passes across the field (Mehta et al., 2000). Expansion is NMDA receptor dependent (Ekstrom et al., 2001) and results primarily from an increase in firing rates at locations preceding the initially symmetric place field. Such an expansion can be accounted for by the potentiating aspect of STDP at CA3-CA1 synapses (Mehta et al., 2000).

The occurrence of LTP when pairings are given five times per second but not at lower rates suggests a requirement for a biochemical signal whose action can be integrated on a timescale of several tenths of a second. In past work, extracellular stimulation protocols for inducing LTP at CA3-CA1 synapses have activated CA1 neurons on a sustained timescale. These protocols include theta-burst stimulation (repeated bursts given at 5-8 Hz) and single prolonged bursts ( $100 \mathrm{~Hz}$ for $1 \mathrm{~s}$ ). Another example of such integration is priming, in which potentiation requires presynaptic activity $200 \mathrm{~ms}$ before a strong paired burst of presynaptic and postsynaptic action potentials (Larson and Lynch, 1986; Rose and Dunwiddie, 1986). One molecular event that can take place on a timescale of tenths of seconds is recovery from potassium channel inactivation, a process that can cumulatively activate dendrites (Hoffman et al., 1997). Another possibility is that, when pairings are repeated at the $5 \mathrm{~Hz}$ frequency, dendrites slowly become more depolarized because of summing of synaptic responses. At synapses between thick tufted layer $\mathrm{V}$ neurons in rat visual cortex, postsynaptic depolarizing currents can relieve a frequency requirement for LTP induction. Other factors might involve calcium and its targets. For instance, CA1 neurons express a slow-equilibrating calcium buffering protein, calbindin. Calbindin could be progressively saturated by a succession of calcium transients such that, in a train of repeated pulses of calcium, later pulses would be more effective in raising calcium concentrations (Maeda et al., 1999). Yet another candidate factor is calmodulin kinase type II (CaMKII), which is strongly activated by repeated pulses of calcium (Lisman et al., 2002). CaMKII is capable of autophosphorylation, a step that makes the enzyme active without the need for calcium. To reach this state, CaMKII may need to first reach a threshold initial level of phosphorylation; the steps leading to this threshold may have the properties of an integrator (Lisman and Spruston, 2005).

Sufficient depolarization to induce potentiation may occur under certain nonbursting conditions. Single postsynaptic spikes are sufficient if they are broadened by filling the neuron with cesium, a potassium channel blocker. Under more natural conditions, single spikes are sufficient to induce potentiation at early ages, when the development of inhibition is incomplete, or when inhibition is blocked by $\mathrm{GABA}_{\mathrm{A}}$ receptor antagonists (Meredith et al., 2003). At other cortical synapses and in hippocampal slice culture, it has been shown that LTP induction may also require the size of EPSPs evoked to be sufficiently strong (Debanne et al., 1996; Sjöström et al., 2001). In contrast, at this synapse, even when EPSPs are suprathreshold for action potential initiation, no potentiation is seen (Fig. 2).

Depression occurs under a wider variety of stimulus conditions than potentiation. It is not strongly influenced by the firing mode of the postsynaptic neuron or the frequency of pairing and can be induced by a broad range of timings of pairs of action potentials. These observations are consistent with the hypothesis that depression is triggered by moderate elevations of calcium (Artola and Singer, 1993). However, depression does show one requirement not seen for potentiation: the need for many pairings extended over seconds. The requirement for prolonged activation to induce LTD compared with LTP has been observed with extracellular stimulation (Dudek and Bear, 1992; Mizuno et al., 2001), pairing of presynaptic and postsynaptic activity (O'Connor et al., 2005a), and calcium uncaging (Yang et al., 1999). All of these lines of evidence indicate that signals that trigger LTD have a longer temporal signature than potentiation signals. One prominent source of prolonged low-frequency activity is slow-wave sleep and quiet wakefulness, during which bursts of activity occur at intervals of $1 \mathrm{~s}$ or longer and repeat over a period of $10 \mathrm{~min}$ or more (Kudrimoti et al., 1999; Lee and Wilson, 2002).

Gating of hippocampal LTP and LTD by behavioral state may have advantages for information storage. This possibility can be seen in computational models that use biologically measured STDP learning rules. In these models, the learning rule is used as a look-up table to guide how much plasticity each pairing contributes to connections in a network of neurons (Song et al., 2000; Rubin et al., 2001). Model networks are therefore capable of both LTP and LTD at all times and are stable only when the statistics of spike timing lead to no net plasticity. At CA3-CA1 synapses, we find that potentiation requires bursts or other strong depolarization. Depression requires extended pairing, thus allowing change to occur only when specific activity patterns occur on longer timescales. The ability of depression and potentiation to be gated at different times provides a means for hippocampal networks to maintain stable connection strengths and stable firing rates (Buzsaki et al., 2002) and may enable associative memory networks to learn correct memories and "unlearn" spuriously stored memories (Hopfield et al., 1983).

The CA3-CA1 synapse is an apt site for modeling synaptic learning rules. Previous models of complex multiple spiketiming-dependent rules have taken a phenomenological approach based on data from pairing spike triplets and quadruplets (Froemke and Dan, 2002) or by increasing the pairing frequency until adjacent pairings fall within the measured timing curve (Sjöström et al., 2001). However, at CA3-CA1 synapses, a superposition-based framework is insufficient in the sense that the nonlinearities are too large. We paired with single postsynaptic action potentials at $0.1-0.5 \mathrm{~Hz}$ and measured a learning rule that was LTD only. It is not possible to reconstruct any plasticity rule that includes LTP from such data.

At CA3-CA1 synapses, for which considerable physiological and molecular information exists, another possible theoretical approach is to model the induction mechanisms that lead to plasticity. Work thus far has focused on calcium dynamics at the synapse resulting from influx through NMDA receptors and voltage-sensitive calcium channels (Shouval et al., 2002; Abarbanel et al., 2005; Rubin et al., 2005). These models can capture the malleability of synaptic learning rules that occurs when activity parameters such as frequency are varied. Future targets of modeling should include spike bursts, the duration of pairings, calcium buffers, and the role of calcium release mechanisms via metabotropic pathways. Consideration of these mechanisms is complemented by our experimental data, which provide a framework for models that capture the full complexity of synaptic learning rules.

\section{References}

Abarbanel HDI, Talathi SS, Gibb L, Rabinovich M (2005) Synaptic plasticity with discrete state synapses. Phys Rev E Stat Nonlin Soft Matter Phys 72:1-14.

Abbott LF, Nelson SB (2000) Synaptic plasticity: taming the beast. Nat Neurosci 3:1178-1183.

Artola A, Singer W (1993) Long-term depression of excitatory synaptic transmission and its relationship to long-term potentiation. Trends Neurosci $16: 480-487$. 
Bell CC, Han VZ, Sugawara Y, Grant K (1997) Synaptic plasticity in a cerebellum-like structure depends on temporal order. Nature 387:278-281.

Bi GQ, Poo M-m (1998) Synaptic modifications in cultured hippocampal neurons: dependence on spike timing, synaptic strength, and postsynaptic cell type. J Neurosci 18:10464-10472.

Blanton MG, LoTurco JJ, Kriegstein AR (1989) Whole cell recording from neurons in slices of reptilian and mammalian cerebral cortex. J Neurosci Methods 30:203-210.

Bliss TVP, Collingridge GL (1993) A synaptic model of memory: long-term potentiation in the hippocampus. Nature 361:31-39.

Buzsaki G (2002) Theta oscillations in the hippocampus. Neuron 33:325-340.

Buzsaki G, Csicsvari J, Dragoi G, Harris K, Henze D, Hirase H (2002) Homeostatic maintenance of neuronal excitability by burst discharges in vivo. Cereb Cortex 12:893-899.

Christie BR, Magee JC, Johnston D (1996) The role of dendritic action potentials and $\mathrm{Ca}^{2+}$ influx in the induction of homosynaptic long-term depression in hippocampal CA1 pyramidal neurons. Learn Mem 3:160-169.

Debanne D, Gähwiler BH, Thompson SH (1996) Cooperative interactions in the induction of long-term potentiation and depression of synaptic excitation between hippocampal CA3-CA1 cell pairs in vitro. Proc Natl Acad Sci USA 93:11225-11230.

Dudek SM, Bear MF (1992) Homosynaptic long-term depression in area $\mathrm{CAl}$ of hippocampus and effects of $\mathrm{N}$-methyl-D-aspartate receptor blockade. Proc Natl Acad Sci USA 89:4363-4367.

Egger V, Feldmeyer D, Sakmann B (1999) Coincidence detection and changes of synaptic efficacy in spiny stellate neurons in rat barrel cortex. Nat Neurosci 2:1098-1105.

Ekstrom AD, Meltzer J, McNaughton BL, Barnes CA (2001) NMDA receptor antagonism blocks experience-dependent expansion of hippocampal "place fields." Neuron 31:631-638.

Feldman DE (2000) Timing-based LTP and LTD at vertical inputs to layer II/III pyramidal cells in rat barrel cortex. Neuron 27:45-56.

Froemke RC, Dan Y (2002) Spike-timing-dependent synaptic modification induced by natural spike trains. Nature 416:433-438.

Froemke RC, Poo MM, Dan Y (2005) Spike-timing-dependent synaptic plasticity depends on dendritic location. Nature 434:221-225.

Gustafsson B, Wigström H, Abraham WC, Huang YY (1987) Long-term potentiation in the hippocampus using depolarizing current pulses as the conditioning stimulus to single volley synaptic potentials. J Neurosci 7:774-780.

Hebb DO (1949) Organization of behavior: a neuropsychological theory. New York: Wiley.

Hoffman DA, Magee JC, Colbert CM, Johnston D (1997) $\mathrm{K}^{+}$channel regulation of signal propagation in dendrites of hippocampal pyramidal neurons. Nature 387:869-875.

Hopfield JJ, Feinstein DI, Palmer RG (1983) “Unlearning" has a stabilizing effect in collective memories. Nature 304:158-159.

Kudrimoti HS, Barnes CA, McNaughton BL (1999) Reactivation of hippocampal cell assemblies: effects of behavioral state, experience, and EEG dynamics. J Neurosci 19:4090-4101.

Larson J, Lynch G (1986) Induction of synaptic potentiation in hippocampus by patterned stimulation involves two events. Science 232:985-988.

Lee AK, Wilson MA (2002) Memory of sequential experience in the hippocampus during slow wave sleep. Neuron 36:1183-1194.

Levy WB, Steward O (1983) Temporal contiguity requirements for longterm associative potentiation/depression in the hippocampus. Neuroscience 8:791-797.

Lisman J, Spruston N (2005) Postsynaptic depolarization requirements for LTP and LTD: a critique of spike timing-dependent plasticity. Nat Neurosci 8:839-841.

Lisman J, Schulman H, Cline H (2002) The molecular basis of CaMKII function in synaptic and behavioural memory. Nat Rev Neurosci 3:175-190.

Maeda H, Ellis-Davies GCR, Ito K, Miyashita Y, Kasai H (1999) Supralinear $\mathrm{Ca}^{2+}$ signaling by cooperative and mobile $\mathrm{Ca}^{2+}$ buffering in Purkinje neurons. Neuron 24:989-1002.

Malenka RC, Nicoll RA (1999) Long-term potentiation: a decade of progress? Science 285:1870-1874.

Malenka RC, Kauer JA, Perkel DJ, Mauk MD, Kelly PT, Nicoll RA, Waxham MN (1989) An essential role for postsynaptic calmodulin and protein kinase activity in long-term potentiation. Nature 340:554-557.

Malinow R, Schulman H, Tsien RW (1989) Inhibition of postsynaptic PKC or CaMKII blocks induction but not expression of LTP. Science 245:862-866.
Markram H, Lübke J, Frotscher M, Sakmann B (1997) Regulation of synaptic efficacy by coincidence of postsynaptic APs and EPSPs. Science 275:213-215.

Mehta MR, Quirk MC, Wilson MA (2000) Experience-dependent asymmetric shape of hippocampal receptive fields. Neuron 25:707-715.

Meredith RM, Floyer-Lea AM, Paulsen O (2003) Maturation of long-term potentiation induction rules in rodent hippocampus: role of GABAergic inhibition. J Neurosci 23:11142-11146.

Mizuno T, Kanazawa I, Sakurai M (2001) Differential induction of LTP and LTD is not determined solely by instantaneous calcium concentration: an essential involvement of a temporal factor. Eur J Neurosci 14:701-708.

Mulkey RM, Herron CE, Malenka RC (1993) An essential role for protein phosphatases in hippocampal long-term depression. Science 261: $1104-1107$.

Nishiyama M, Hong K, Mikoshiba K, Poo M-m, Kato K (2000) Calcium stores regulate the polarity and input specificity of synaptic modification. Nature 408:584-588.

O'Connor DH, Wittenberg GM, Wang SS-H (2005a) Graded bidirectional synaptic plasticity is composed of switch-like unitary events. Proc Natl Acad Sci USA 102:9679-9684.

O'Connor DH, Wittenberg GM, Wang SS-H (2005b) Dissection of bidirectional synaptic plasticity into saturable unidirectional processes. J Neurophysiol 94:1565-1573.

Otto T, Eichenbaum H, Wiener SI, Wible CG (1991) Learning-related patterns of CA1 spike trains parallel stimulation parameters optimal for inducing hippocampal long-term potentiation. Hippocampus 1:181-192.

Paulsen O, Sejnowski TJ (2000) Natural patterns of activity and long-term synaptic plasticity. Curr Opin Neurobiol 10:172-179.

Pike FG, Meredith RM, Olding AW, Paulsen O (1999) Rapid report: postsynaptic bursting is essential for "Hebbian" induction of associative long-term potentiation at excitatory synapses in rat hippocampus. J Physiol (Lond) 518:571-576.

Rose GM, Dunwiddie TV (1986) Induction of hippocampal long-term potentiation using physiologically patterned stimulation. Neurosci Lett 69:244-248.

Rubin J, Lee DD, Sompolinsky H (2001) Equilibrium properties of temporally asymmetric Hebbian plasticity. Phys Rev Lett 86:364-367.

Rubin JE, Gerkin RC, Bi GQ, Chow CC (2005) Calcium time course as a signal for spike-timing-dependent plasticity. J Neurophysiol 93:2600-2613.

Sanes JR, Lichtman JW (1999) Can molecules explain long-term potentiation? Nat Neurosci 2:597-604.

Shouval HZ, Bear MF, Cooper LN (2002) A unified model of NMDA receptor-dependent bidirectional synaptic plasticity. Proc Natl Acad Sci USA 99:10831-10836.

Sjöström PJ, Turrigiano GG, Nelson SB (2001) Rate, timing, and cooperativity jointly determine cortical synaptic plasticity. Neuron 32:1149-1164.

Song S, Miller KD, Abbott LF (2000) Competitive Hebbian learning through spike-timing-dependent synaptic plasticity. Nat Neurosci 3:919-926.

Steele PM, Mauk MD (1999) Inhibitory control of LTP and LTD: stability of synapse strength. J Neurophysiol 81:1559-1566.

Stuart G, Schiller J, Sakmann B (1997) Action potential initiation and propagation in rat neocortical pyramidal neurons. J Physiol (Lond) 505:617-632.

Tsubokawa H, Ross WN (1996) IPSPs modulate spike backpropagation and associated $\left[\mathrm{Ca}^{2+}\right]_{\mathrm{i}}$ changes in the dendrites of hippocampal CA1 pyramidal neurons. J Neurophysiol 76:2896-2906.

Tsukada M, Aihara T, Kobayashi Y, Shimazaki H (2005) Spatial analysis of spike-timing-dependent LTP and LTD in the CA1 area of hippocampal slices using optical imaging. Hippocampus 15:104-109.

Wang HX, Gerkin RC, Nauen DW, Bi GQ (2005) Coactivation and timingdependent integration of synaptic potentiation and depression. Nat Neurosci 8:187-193.

Wang SS-H, Denk W, Häusser M (2000) Coincidence detection in single dendritic spines mediated by calcium release. Nat Neurosci 3:1266-1273.

Watanabe S, Hoffman DA, Migliore M, Johnston D (2002) Dendritic $\mathrm{K}^{+}$ channels contribute to spike-timing dependent long-term potentiation in hippocampal pyramidal neurons. Proc Natl Acad Sci USA 99:8366-8371.

Williams SR, Stuart GJ (1999) Mechanisms and consequences of action potential burst firing in rat neocortical pyramidal neurons. J Physiol (Lond) 521:467-482.

Yang SN, Tang YG, Zucker RS (1999) Selective induction of LTP and LTD by postsynaptic $\left[\mathrm{Ca}^{2+}\right]_{\mathrm{i}}$ elevation. J Neurophysiol 81:781-787. 